\title{
8 Antenna Polar Switching Up-Down Relay Networks
}

\author{
Jun $\mathrm{Li}^{1} \cdot$ Moon $\mathrm{Ho} \mathrm{Lee}^{2} \cdot$ Yier $\mathrm{Yan}^{3} \cdot \mathrm{Bu}$ Shi Peng ${ }^{4} \cdot$ Gun Joon Hwang ${ }^{5}$
}

\begin{abstract}
In this paper, we propose a reliable $8 \times 8$ up-down switching polar relay code based on 3 GPP LTE standard, motivated by 3 GPP LTE down link, which is $30 \mathrm{bps} / \mathrm{Hz}$ for $8 \times 8$ MIMO antennas, and by Arikan's channel polarization for the frequency selective fading (FSF) channels with the generator matrix $Q_{8}$. In this scheme, a polar encoder and OFDM modulator are implemented sequentially at both the source node and relay nodes, the time reversion and complex conjugation operations are separately implemented at each relay node, and the successive interference cancellation (SIC) decoder, together with the cyclic prefix (CP) removal, is performed at the destination node. Use of the scheme shows that decoding at the relay without any delay is not required, which results in a lower complexity. The numerical result shows that the system coded by polar codes has better performance than currently used designs.
\end{abstract}

Key words: Polar Code, Up-Down Relay Network, SIC Decoder.

\section{Introduction}

Channel polarization shows a construction of provably capacity-achieving coding sequences with belief propagation (BP) decoders [1], [2]. It provides a potential method to meet the elusive goal of multi-fold binaryinput discrete memoryless channels, where channel combining and splitting operations are applied to improve its symmetric capacity [1]. In actuality, the polarization of multiple channels is a commonplace phenomenon and thus almost impossible to avoid as long as several channels are synthesized in a proper density with certain arrangements. During the past decade, the space time coding (STC) communication system has been well studied as a promising way to increase spectral efficiency, channel capacity and link reliability [4] [7]. It shows that the coding gain and diversity can be simultaneously achieved with suitable coding schemes. The problem with the relay schemes is the data rate loss that occurs as the number of relay nodes increases, since relay nodes are assumed to be assigned orthogonal channels. This leads to the use of polar coding sequences in relay channels, where relay nodes are allowed to transmit simultaneously over the same channels.

In this paper, we consider a simple design of the relay system that achieves the fascinating symmetric capacity of the relay channels based on polar coding with a belief propagation (BP) decoder at destination node. The paper is organized as follows. In Section II, we describe the state switching Up-Down polar Relay System and the capacity at the source and the each relay. In Section III, we systematically decode the design of a simple polar relay scheme for up-down polar relay channels by using a successive cancellation structure. Some simulation results are also depicted in order to show the BER performance behavior and robustness of this polar relay system. Finally, conclusions are drawn in Section IV.

\section{II . Conventional Polar Code}

In Arikan's paper [1], a polar code is proposed that is a class of capacity-achieving codes. The main motivation of polar code was theoretical to show the existence of a family of codes that are provably capacity achieving and that have low complexity encoding and decoding algorithms.

Polar code is the first provably capacity achieving code for arbitrary B-DMC W with low encoding and decoding complexity. The polar codes are constructed to transform $Q_{2}^{\otimes 3}$ to a block of $N=2^{n}$ bits and transmit the output through independent copies of a B-DMC where ' $\otimes$ ' denotes Kronecker product. As $\mathrm{n}$ grows large, the channels seen by individual bits start polarizing: they approach either a noiseless channel or a pure-noise cha-

\footnotetext{
Manuscript received June 22, 2011 ; revised October 13, 2011. (ID No. 20110622-019J)

${ }^{1}$ Div. of Electronic \& Information Engineering, Chonbuk National University, Jeonju, Korea.

${ }^{2}$ Div. of Electronic Engineering, Chonbuk National University, Jeonju, Korea.

${ }^{3}$ Mechanical and Electronic Engineering, Guangzhou University, Guangzhou, P. R. China.

${ }^{4}$ Div. of Electronic \& Information Engineering, Chonbuk National University, Jeonju, Korea.

${ }^{5}$ Supporting Center of Information \& Communication, Jeonju University, Jeonju, Korea.

Corresponding Author : Moon Ho Lee (e-mail : moonho@jbnu.ac.kr)
} 
nnel, where the fraction of channels becoming noiseless is close to the symmetric mutual information $\mathrm{I}(\mathrm{W})$ that presents high rates in reliable wireless communication channel as inputs of $\mathrm{W}$ with equal frequencies.

Constructions of polar codes of order $2^{n}$ are derived from radix $2^{\mathrm{n}}$ channel polarization, which is an operation whereby one manufacture out of $\mathrm{N}$ independent copies of a given $\mathrm{BDMC} \mathrm{W}$ for $\mathrm{N}=2^{\mathrm{n}}$ yields a second set of $N$ channels $\mathrm{W}_{N}^{i}: 1 \leq i \leq N$ that show a polarization effect in the sense that, as $N$ becomes large, the symmetric capacity terms $I\left(\mathrm{~W}_{N}^{i}\right): 1 \leq i \leq \mathrm{N}$ tend towards 0 or 1 for all but a vanishing fraction of indices $i$. This operation consists of a channel-combining phase and a channel-splitting phase. This phase combines copies of a given $\mathrm{B}-\mathrm{DMC}$ in a recursive manner to produce a vector channel $W_{N}$ for $\mathrm{N}=2^{\mathrm{n}}$. The recursion begins with only one copy of $\mathrm{W}$ and we set $\mathrm{W}_{1}=\mathrm{W}$ The first level of the recursion combines two independent copies, as shown in Fig. 1, and obtains the channel $W_{2}$ with the transition probabilities:

$$
\mathrm{W}_{2}\left(y_{1}^{2} \mid u_{1}^{2}\right)=\mathrm{W}\left(y_{1} \mid \otimes_{i=1}^{2} u_{i}\right) \mathrm{W}\left(y_{2} \mid u_{2}\right)
$$

where $\otimes_{i=1}^{2} u_{i}$. The mapping $\mathrm{W}_{2}: u_{1}^{2} \rightarrow y_{1}^{2}$ from input of $\mathrm{W}_{2}$ can be denoted by:

$$
\mathrm{W}_{2}\left(y_{1}^{2} \mid u_{1}^{2}\right)=\mathrm{W}^{2}\left(y_{1}^{2} \mid u_{1}^{2} G_{2}\right)
$$

where

$$
G_{2}=\left(\begin{array}{ll}
1 & 0 \\
1 & 1
\end{array}\right)
$$

The mapping $\mathrm{W}_{4}: u_{1}^{4} \rightarrow y_{1}^{4}$ from the input of $\mathrm{W}_{4}$ to the output of $\mathrm{W}_{4}$, and hence the next level of the recursion, is shown in Fig. 2, where four independent copies of $\mathrm{W}_{2}$ are combined to create the channel $\mathrm{W}_{22}$ with transition probabilities denoted by:

$$
\begin{aligned}
& \mathrm{W}_{4}\left(y_{1}^{4} \mid u_{1}^{4}\right)=\mathrm{W}^{4}\left(y_{1}^{4} \mid u_{i}^{4} G_{4}\right) \\
& =\mathrm{W}_{2}\left(y_{1}^{2} \mid \otimes_{i=2}^{2} u_{i}, \otimes_{i=3}^{4} u_{i}\right) \mathrm{W}_{2}\left(y_{3}^{4} \mid u_{2}, u_{4}\right)
\end{aligned}
$$

where

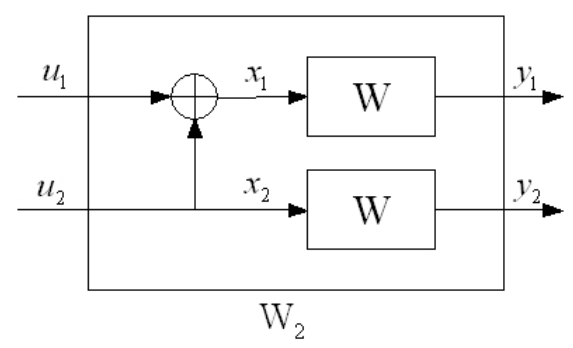

Fig. 1 . The 2 by 2 Arikan polar code.

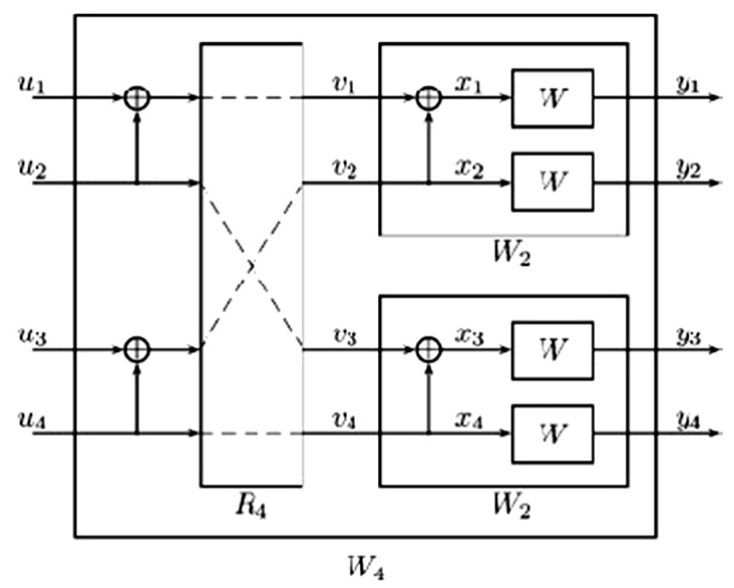

Fig. 2. The 4 by 4 Arikan polar code.

$$
G_{4}=R_{4} G_{2}^{\otimes 2}=\left(\begin{array}{cccc}
1 & 0 & 0 & 0 \\
1 & 1 & 0 & 0 \\
1 & 0 & 1 & 0 \\
1 & 1 & 1 & 1
\end{array}\right)
$$

Note that $R_{4}\left(u_{1}^{4}\right)=\left(u_{1}, u_{2}, u_{3}, u_{4}\right)$.

The general form of the recursion is specified where four independent copies of $\mathrm{W}_{N / 2}$ are combined to produce the channel $\mathrm{WN}_{\mathrm{N}}$ for $\mathrm{N}=2 \mathrm{n}$. The mapping $u_{1}^{N} \rightarrow y_{1}^{N}$ from the input of the synthesized channel to the input of the underlying raw channels is clearly linear over GF (2).

Thus, it is represented by a matrix $G(N)$, such that

$$
\mathrm{W}_{N}\left(y_{1}^{N} \mid u_{1}^{N}\right)=\mathrm{W}^{N}\left(y_{1}^{N} \mid u_{1}^{N} G_{N}\right)
$$

where $y_{1}^{N} \in \eta_{1}^{N}, u_{1}^{N} \in \chi^{N}$, and $G^{N}=G_{2}^{\otimes n}$.

The $2 \times 2$ and $4 \times 4$ polar codes are shown in Fig. 1 and Fig. 2, respectively. As shown in [1], asymptotically, the polar codes as a function of code block length $\mathrm{N}$, can be encoded in complexity $O(\mathrm{~N} \log N)$, and decoded using a successive cancellation decoder in complexity $O(\mathrm{~N} \log N)$. The polar code can be used in a MIMO network system.

\section{Proposed Channel Polarization: Up-Down-Polar Relay System}

In [3], the authors presented a MIMO system that employed reconfigurable antennas that were used as both transmitter and receiver parts. This MIMO system has a good diversity gain and multiplexing gain, as shown in Table 1. As the number of antennas increase, the diversity gain and multiplexing gain also increase proportionally. This is why we consider 8 antennas in our proposed scheme.

As Fig. 3 shows, we can easily see that the multiple 
Table 1. Error probability and channel capacity with different number of antennas.

\begin{tabular}{|c|c|c|}
\hline $\begin{array}{c}\text { Number of } \\
\text { antennas }\end{array}$ & $\begin{array}{c}\text { Error } \\
\text { probability }\left(P_{e}\right)\end{array}$ & $\begin{array}{c}\text { Capacity }(C), \\
\text { bps } / \mathrm{H}_{z}\end{array}$ \\
\hline $\begin{array}{c}N_{s}=N_{r}=1 \\
\text { (SISO) }\end{array}$ & $P \propto S N R^{-1}$ & $C=\log (\mathrm{SNR})$ \\
\hline $\begin{array}{c}N_{s}=1, N_{r}>1 \\
(\mathrm{SIMO})\end{array}$ & $P \propto S N R^{-N_{r}}$ & $C=\log (\mathrm{SNR})$ \\
\hline $\begin{array}{c}N_{s}>1, N_{r}>1 \\
(\mathrm{MIMO})\end{array}$ & $\begin{array}{c}P \propto S N R^{-N_{t} N_{r}} \\
N_{t} N_{r}=\text { Diversity gain }\end{array}$ & $\begin{array}{c}C=\min \left(N_{t}, N_{r}\right) \log (\mathrm{SNR}) \\
\text { gain min }\left(N_{t}, N_{r}\right)= \\
\text { spectral multiplexing }\end{array}$ \\
\hline
\end{tabular}

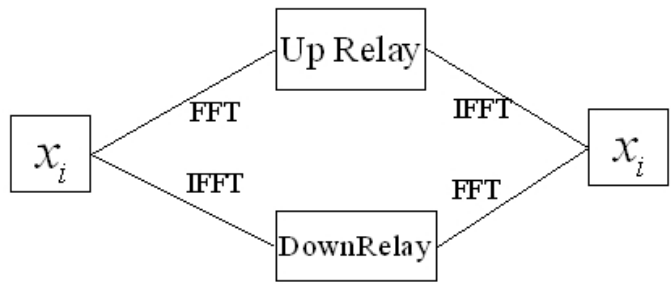

Fig. 3. Up-Down relay network model.

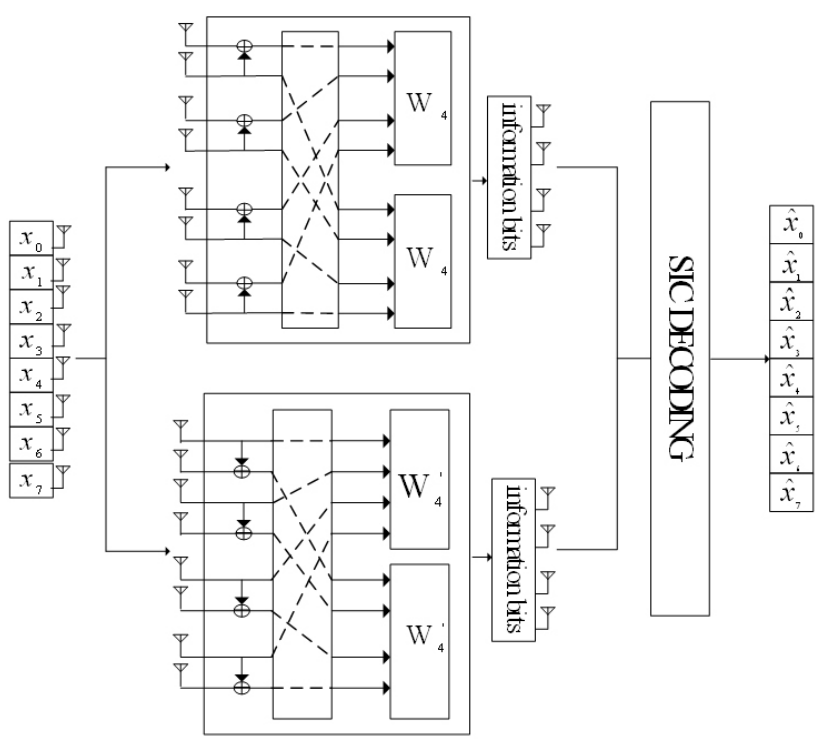

Fig. 4. Proposed 8 antenna polar switching up-down relay networks.

antenna system has a higher diversity gain and multiplexing gain. We therefore consider a distributed wireless system based on OFDM modulation with $\mathrm{N}$ subcarriers. The system has one source node $\mathrm{S}$, one destination node $\mathrm{D}$, and two relay nodes $R \triangleq\left\{R_{1}, R_{2}\right\}$, as shown in Fig. 4. The numbers of antennas at $\mathrm{S}, \mathrm{R}$, and $\mathrm{D}$ are $\mathrm{N}_{\mathrm{s}}, \mathrm{N}_{\mathrm{r}}$, and $\mathrm{N}_{\mathrm{d}}$, respectively. We consider a scenario where $\mathrm{N}_{\mathrm{s}}$ OFDM symbols are transmitted for $\mathrm{N}_{\mathrm{s}}=2^{\mathrm{n}}$. We consider a design of the polar relay scheme that can mitigate relay synchronization errors. Each relay node, $R_{k}, \forall k \in\{1,2\}$, is assumed to be capable of processing the OFDM symbols independently and correctly. The average transmission power at source node $\mathrm{S}$ is $\mathrm{p}_{\mathrm{t}}$. In this paper, we let $\mathrm{N}_{\mathrm{s}}=\mathrm{N}_{\mathrm{r}}=\mathrm{N}_{\mathrm{d}}=1$. This assumption is applicable for any nodes equipped with multiple antennas. The relay scheme is half-duplex, meaning that $\mathrm{S}$ and $\mathrm{R}$ do not transmit and receive simultaneously. The $\mathrm{N}_{\mathrm{s}}$ independent OFDM symbols are transmitted simultaneously from source node $\mathrm{S}$ to destination node $\mathrm{D}$ in two stages. The constraint on the total network power is $p=p_{t}+2 p_{r}$. We also adopt the power allocation strategy suggested in [3], and thus have

$$
p_{t}=2 p_{r}=p / 2
$$

This channel model, denoted by $\mathrm{H} \in C^{2 \times 2}$, is created between source node $\mathrm{S}$ and relay nodes $\mathrm{R}$, and between $\mathrm{K} \in C^{2 \times 2}$ and $\mathrm{D}$. Here, entries of $\mathrm{H}$ and $\mathrm{K}$ are assumed independent and identically distributed (i.i.d.) with distribution $\mathrm{CN}(0,1)$.

The relay wireless system with source-relay-destination triplet structure is equivalent to two partial wireless systems. The polar channel model is shown as:

$$
\begin{aligned}
& \text { up polar channel }: \mathrm{H}=\operatorname{diag}\left(\phi_{1}, \phi_{2}\right), \\
& \text { down polar channel: } K=\operatorname{diag}\left(\kappa_{1}, \kappa_{2}\right) .
\end{aligned}
$$

where the entries $\phi_{k}$ and $\kappa_{k}$ are independent complex Gaussian random variables with zero-mean and unitvariance.

Based on the MIMO relay channels $\mathrm{H}$ and $\mathrm{K}$ in (8) and (9), respectively, we designed the polar system for the transmission of the signal vector $\mathrm{x}$, in which we switch to the polar system for four time slots; i.e., up-downpolarizing MIMO relay communication models in a turning switch.

The system has two phases, as follows. In phase 1, the source node broadcasts the information OFDM symbols that are polarized at source node $\mathrm{S}$ to each relay node $\mathrm{R}_{\mathrm{k}}$. In phase $2, \mathrm{~S}$ stops the transmission and each relay node $R_{k}$ that has polarized the received OFDM symbols now processes and simultaneously retransmits the resulting symbols to destination node D.

\section{Up-Down State Switching Polarizing Cooperative Relay System}

At source node $\mathrm{S}$, the transmitted information is modulated into complex symbols $x_{i j}$ and then each $\mathrm{N}$ modulated symbol as a block is poured into an OFDM modulator of $\mathrm{N}$ subcarriers. We denote four consecutive OFDM blocks by $x_{i}=\left(x_{i, 0}, x_{i, 1}, \cdots, x_{i, N-1}\right)^{\mathrm{T}}, \forall i \in Z_{8}$. We define $\left(x_{i}+x_{j}\right)=\left(x_{i, 0}+x_{j, 0}, x_{i, 1}+x_{j, 1}, \cdots, x_{i, N-1}+x_{j, N-1}\right)^{T}, \forall i, j \in Z_{8}$, for calculation.

In the first time slot, the eight consecutive OFDM 
blocks are processed with the up-polarizing $8 \times 8$ matrix $Q_{8}$ at source node $\mathrm{S}$, i.e.,

$$
\mathrm{U}=Q_{8} \mathrm{X}
$$

where $U=\left(u_{0}, u_{1}, u_{2}, u_{3}, u_{4}, u_{5}, u_{6}, u_{7}\right)$ denotes the polarized matrix of size $N \times 8, X=\left(x_{0}, x_{1}, x_{2}, x_{3}, x_{4}, x_{5}, x_{6}, x_{7}\right)$ denotes the signal matrix of size $N \times 8$ corresponding to eight OFDM blocks, $Q_{8}=Q_{2} \otimes Q_{2} \otimes Q_{2}=Q_{2}^{\otimes 3}$, which is given as:

$$
\text { up }: Q_{2}=\left(\begin{array}{ll}
1 & 0 \\
1 & 1
\end{array}\right), \text { down }: Q_{2}^{T}=\left(\begin{array}{ll}
1 & 1 \\
0 & 1
\end{array}\right)
$$

The following indicates the up switch:

$$
Q_{8}=\left(\begin{array}{llllllll}
1 & 0 & 0 & 0 & 0 & 0 & 0 & 0 \\
1 & 1 & 0 & 0 & 0 & 0 & 0 & 0 \\
1 & 0 & 1 & 0 & 0 & 0 & 0 & 0 \\
1 & 1 & 1 & 1 & 0 & 0 & 0 & 0 \\
1 & 0 & 0 & 0 & 1 & 0 & 0 & 0 \\
1 & 1 & 0 & 0 & 1 & 1 & 0 & 0 \\
1 & 0 & 1 & 0 & 1 & 0 & 1 & 0 \\
1 & 1 & 1 & 1 & 1 & 1 & 1 & 1
\end{array}\right) .
$$

and the down of the transpose of the above matrix is

$$
Q_{8}^{T}=\left(\begin{array}{llllllll}
1 & 1 & 1 & 1 & 1 & 1 & 1 & 1 \\
0 & 1 & 0 & 1 & 0 & 1 & 0 & 1 \\
0 & 0 & 1 & 1 & 0 & 0 & 1 & 1 \\
0 & 0 & 0 & 1 & 0 & 0 & 0 & 1 \\
0 & 0 & 0 & 0 & 1 & 1 & 1 & 1 \\
0 & 0 & 0 & 0 & 0 & 1 & 0 & 1 \\
0 & 0 & 0 & 0 & 0 & 0 & 1 & 1 \\
0 & 0 & 0 & 0 & 0 & 0 & 0 & 1
\end{array}\right) .
$$

In the OFDM modulator, the eight consecutive blocks are modulated by N-point FFT. Each block is then precoded by a cyclic prefix (CP) with length $l_{c p}$. Thus, each OFDM symbol consists of $L_{s}=N+l_{c p}$ samples. Finally, the four OFDM symbols are broadcasted to two relay nodes denoted by $\tau_{s d 2}$, the overall relative delay from source node $\mathrm{S}$ to relay node $\mathrm{R}_{2}$, and then to destination node $\mathrm{D}$, where the delay is relative to relay node $\mathrm{R} 1$. In order to combat against both frequency selective fading channels and timing errors, we assume that $l_{c p} \geq \max _{l, k}\left\{\tau_{l, s k}+\tau_{l, r k}+\tau_{s d 2}\right\}$. We denote eight consecutive OFDM symbols by $\breve{u}_{i}, \forall i \in Z_{8}$, where $\breve{u}_{i}$ consists of $\operatorname{FFT}\left(u_{i}\right)$ and the corresponding CP.

At each relay $R_{k}$, the received noisy signals will be simply processed and forwarded to destination node D. We assume that the channel coefficients are constant during eight OFDM symbol intervals. We define two processed vectors $\breve{\mathrm{u}}_{1}=\left(\breve{u}_{0}^{T}, \breve{u}_{1}^{T}, \breve{u}_{2}^{T}, \breve{u}_{3}^{T}\right)^{T}$ and $\breve{\mathrm{u}}_{2}=\left(\breve{u}_{4}^{T}, \breve{u}_{5}^{T}, \breve{u}_{6}^{T}, \breve{u}_{7}^{T}\right)^{T}$, which are polarized at $R_{1}$ and $R_{2}$, respectively.

Therefore, the received signals at $R_{k}, \forall k \in\{1,2\}$, for eight successive OFDM symbol durations can be generated as:

$$
\begin{aligned}
& \breve{r}_{k 0}=\sqrt{p_{t}} \breve{u}_{0} * \phi_{k}+\breve{n}_{k 0}, \\
& \breve{r}_{k 1}=\sqrt{p_{t}} \breve{u}_{1} * \phi_{k}+\breve{n}_{k 1}, \\
& \breve{r}_{k 2}=\sqrt{p_{t}} \breve{u}_{2} * \phi_{k}+\breve{n}_{k 2}, \\
& \breve{r}_{k 3}=\sqrt{p_{t}} \breve{u}_{3} * \phi_{k}+\breve{n}_{k 3}, \\
& \breve{r}_{k 4}=\sqrt{p_{t}} \breve{u}_{4} * \phi_{k}+\breve{n}_{k 4}, \\
& \breve{r}_{k 5}=\sqrt{p_{t}} \breve{u}_{5} * \phi_{k}+\breve{n}_{k 5}, \\
& \breve{r}_{k 6}=\sqrt{p_{t}} \breve{u}_{6} * \phi_{k}+\breve{n}_{k 6}, \\
& \breve{r}_{k 7}=\sqrt{p_{t}} \breve{u}_{7} * \phi_{k}+\breve{n}_{k 7} .
\end{aligned}
$$

where $\sqrt{p_{t}}$ is the transmission power at source node $\mathrm{S}$, $\phi_{k}$ is an $L \times 1$ vector defined as $\phi_{k}=\left(\alpha_{s k}(0), \alpha_{s k}(1), \cdots\right.$, $\left.\alpha_{s k}(L-1)\right), *$ denotes the linear convolution, and $\breve{n}_{k i}, \forall i$ $\in Z_{8}$, denotes the corresponding additive white Gaussian noise(AWGN) at relay node $R_{k}$ with zero-mean and unit-variance, in four successive OFDM symbol durations.

Each relay node $\mathrm{R}_{\mathrm{k}}$ then polarizes, processes, and forwards the received noisy signals $u_{i}, \forall i \in\{1,2, \cdots, 8\}$. After performing the processing operations, each relay node $R_{k}$ amplifies the yielded symbols with a scalar $\lambda=\sqrt{p_{r} / p_{t}+1}$ while retaining the average transmission power $p_{r}$. In order to make the PF scheme available for frequency selective fading channels, each relay $R_{k}$ can only implement the time reversal operations or the complex conjugation operations on the received OFDM symbols.

At destination node $\mathrm{D}$, the $\mathrm{CP}$ is removed for each OFDM symbol. We note that relay node R1 implements the time reversions of the noisy signals including both information symbols and CP. What we need is to obtain the time reversal version of only information symbols, i.e., $\zeta\left(F F T\left(u_{i}\right)\right), \forall i \in Z_{8}$, after the $\mathrm{CP}$ removal. Then, by using some properties of FFT/IFFT, we achieve the feasibility of the definition as follows.

Definition 2.1 Consider the processed OFDM symbols at relay node $R_{1}$ we can obtain

$$
\zeta\left(F F T\left(u_{i}\right)\right) * \zeta\left(\phi_{1}^{\prime}\right)
$$

at destination node if we remove the $\mathrm{CP}$ as in a conventional OFDM system, to get an $\mathrm{N}$-point vector and shift the last $\tau_{1}^{\prime}=l_{c p}-\tau_{1}+1$ samples of the N-point vector as the first $\tau_{1}^{\prime}$ samples. Here $\phi_{1}^{\prime}$ is an $N \times 1$ vector defined as $\phi_{1}^{\prime}=\left(\alpha_{s 1}(0), \cdots, \alpha_{s 1}(L-1), 0, \cdots, 0\right)$, and $\tau_{1}$ denotes the maximum path delay of channel $\phi_{1}$ from source node $S$ to relay node $\mathrm{R} 1$, i.e., $\tau_{1}=\max _{l}\left\{\tau_{l, s 1}\right\}$. In a similar way, we 
define another $N \times 1$ vector $\kappa_{1}^{\prime}$ as $\kappa_{1}^{\prime}=\left(\alpha_{r 1}(0), \cdots, \alpha_{r 1}(L-1)\right.$, $0, \cdots, 0)$.

The received OFDM symbols are then transformed by the N-point FFT [6], [8]. As mentioned before, because of the timing errors, the OFDM symbols from relay node $\mathrm{R} 2$ arrive at destination node $\tau_{s d 2}$ samples later than the symbols from relay node R1. Since $l_{c p}$ is long enough, we can still maintain the orthogonality between subcarriers. The delay $\tau_{s d 2}$ in the time domain corresponds to a phase change in the frequency domain; i.e.:

$$
f^{\tau_{s d 2}}=\left(1, e^{-l 2 \pi \tau_{s d 2} / N}, \cdots, e^{-t 2 \pi \tau_{s d 2}(N-1) / N}\right)^{T},
$$

where $f=\left(1, e^{-t 2 \pi \tau_{s d 2} / N}, \cdots, e^{-l 2 \pi(N-1) / N}\right)^{T}$ and $\imath=\sqrt{-1}$. Similarly, the shift of $\tau_{1}^{\prime}$ samples in the time domain also corresponds to a phase change $f^{\tau_{1}^{\prime}}$, and hence the total phase change is $f^{\tau_{2}}$. We denote $\breve{y}_{i}=\left(\breve{y}_{i 0}, \breve{y}_{i 1}, \cdots, \breve{y}_{i(N-1)}\right)$, $\forall i \in Z_{8}$, the received signals for four consecutive OFDM blocks at destination node $D$ after the $\mathrm{CP}$ removal and the FFT transformations. Consequently, we have

$$
\begin{aligned}
& \breve{y}_{0}=\lambda\left[\sqrt{p_{t}} F F T\left(\zeta\left(F F T\left(u_{0}\right)\right)\right) \circ \breve{\phi}_{1} \circ \breve{\kappa}_{1}+\breve{\bar{n}}_{10} \circ \breve{\kappa}_{1}\right]+\breve{n}_{0} \\
& \breve{y}_{1}=\lambda\left[\sqrt{p_{t}} F F T\left(\zeta\left(F F T\left(u_{1}\right)\right)\right) \circ \breve{\phi}_{1} \circ \breve{\kappa}_{1}+\left(\breve{n}_{10}+\breve{n}_{20}\right) \circ \breve{\kappa}_{1}\right]+\breve{n}_{1} \\
& \breve{y}_{2}=\lambda\left[\sqrt{p_{t}} F F T\left(\zeta\left(F F T\left(u_{0}+u_{2}\right)\right)\right) \circ f^{\tau_{2}} \circ \breve{\phi}_{2} \circ \breve{\kappa}_{2}+\breve{n}_{21} \circ \breve{\kappa}_{2}\right]+\breve{n}_{2} \\
& \breve{y}_{3}=\lambda\left[\sqrt{p_{t}} F F T\left(\zeta\left(F F T\left(u_{1}+u_{3}\right)\right)\right) \circ f^{\tau_{2}} \circ \breve{\phi}_{2} \circ \breve{\kappa}_{2}\right. \\
& \left.+\left(\overline{\bar{n}}_{21}^{*}+\overline{\bar{n}}_{23}^{*}\right) \circ \breve{\kappa}_{2}\right]+\breve{n}_{3} \\
& \breve{y}_{4}=\lambda\left[\sqrt{p_{t}} F F T\left(\zeta\left(F F T\left(u_{4}\right)^{*}\right)\right) \circ \breve{\phi}_{5} \circ \breve{\kappa}_{5}+\bar{n}_{50} \circ \breve{\kappa}_{5}\right]+\breve{n}_{4} \\
& \breve{y}_{5}=\lambda\left[\sqrt{p_{t}} F F T\left(\zeta\left(F F T\left(u_{4}+u_{5}\right)^{*}\right)\right) \circ \breve{\phi}_{5} \circ \breve{\kappa}_{5}+\left(\breve{n}_{50}+\breve{n}_{60}\right) \circ \breve{\kappa}_{5}\right]+\breve{n}_{5} \\
& \breve{y}_{6}=\lambda\left[\sqrt{p_{t}} F F T\left(\zeta\left(F F T\left(u_{4}+u_{6}\right)^{*}\right)\right) \circ f^{\tau_{6}} \circ \breve{\phi}_{6} \circ \breve{\kappa}_{6}+\bar{n}_{61} \circ \breve{\kappa}_{6}\right]+\breve{n}_{6} \\
& \breve{y}_{7}=\lambda\left[\sqrt{p_{t}} F F T\left(\zeta\left(F F T\left(u_{4}+u_{5}+u_{6}+u_{7}\right)^{*}\right)\right) \circ f^{\tau_{6}} \circ \breve{\phi}_{6} \circ \breve{\kappa}_{6}\right. \\
& \left.+\left(\breve{\bar{n}}_{61}^{*}+\overline{\bar{n}}_{63}^{*}\right) \circ \breve{\kappa}_{6}\right]+\breve{n}_{7}
\end{aligned}
$$

where $\breve{\phi}_{1}=F F T\left(\zeta\left(\phi_{1}^{\prime}\right)\right), \quad \breve{\kappa}_{1}=F F T\left(\kappa_{1}^{\prime}\right), \breve{\phi}_{2}=F F T\left(\left(\phi_{1}^{\prime}\right)^{*}\right)$, $\breve{\kappa}_{2}=F F T\left(\kappa_{2}^{\prime}\right), \breve{\bar{n}}_{k i}=F F T\left(\bar{n}_{k i}\right)$, and $\overline{\bar{n}}_{k i}=F F T\left(\bar{n}_{k i}\right), \forall k \in$ and $\{1, \cdots, 8\} \forall i \in Z_{8}$.

\section{4-1 The Up Polar Relay Case}

For the up-polar relay case, we define the new mapping method at the relay from the 2 by 2 up-polar code:

$$
\begin{aligned}
& u_{0}=x_{0}, \\
& u_{1}=x_{0}+x_{1}, \\
& u_{2}=x_{2}, \\
& u_{3}=x_{2}+x_{3}, \\
& u_{4}=x_{0}+x_{4}, \\
& u_{5}=x_{1}+x_{5}, \\
& u_{6}=x_{2}+x_{6}, \\
& u_{7}=x_{3}+x_{7} .
\end{aligned}
$$

According to the properties of the well-known FFT transforms for an $N \times 1$ point vector $\mathrm{x}$, we have $(F F T(x))^{*}$ $=\operatorname{IFFT}\left(x^{*}\right)$ and $\operatorname{FFT}(\zeta(F F T(x)))=\operatorname{IFFT}(F F T(x))=x$. Therefore, the formulas in (16) can be written in the polar code form on each subcarrier $u, \forall u \in Z_{N}$, as follows:

$$
\left(\begin{array}{l}
y_{0 u} \\
y_{1 u} \\
y_{2 u} \\
y_{3 u} \\
y_{4 u} \\
y_{5 u} \\
y_{6 u} \\
y_{7 u}
\end{array}\right)=\left(\begin{array}{cccccccc}
\breve{\phi}_{1} \widetilde{\kappa}_{1} & 0 & 0 & 0 & 0 & 0 & 0 & 0 \\
0 & \breve{\phi}_{1} \widetilde{\kappa}_{1} & 0 & 0 & 0 & 0 & 0 & 0 \\
0 & 0 & \breve{\phi}_{1} \breve{\kappa}_{1} & 0 & 0 & 0 & 0 & 0 \\
0 & 0 & 0 & \breve{\phi}_{1} \breve{\kappa}_{1} & 0 & 0 & 0 & 0 \\
0 & 0 & 0 & 0 & \Phi_{2} & 0 & 0 & 0 \\
0 & 0 & 0 & 0 & 0 & \Phi_{2} & 0 & 0 \\
0 & 0 & 0 & 0 & 0 & 0 & \Phi_{2} & 0 \\
0 & 0 & 0 & 0 & 0 & 0 & 0 & \Phi_{2}
\end{array}\right)\left(\begin{array}{c}
u_{0} \\
u_{1} \\
u_{0}+u_{2} \\
u_{1}+u_{3} \\
u_{4}^{*} \\
u_{4}^{*}+u_{5}^{*} \\
u_{4}^{*}+u_{6}^{*} \\
u_{4}^{*}+u_{5}^{*}+u_{6}^{*}+u_{7}^{*}
\end{array}\right)+e_{0}
$$

$$
\left(\begin{array}{l}
y_{0 u} \\
y_{1 u} \\
y_{2 u} \\
y_{3 u} \\
y_{4 u}^{*} \\
y_{5 u}^{*} \\
y_{6 u}^{*} \\
y_{7 u}^{*}
\end{array}\right)=\left(\begin{array}{cccccccc}
\breve{\phi}_{1} \breve{\kappa}_{1} & 0 & 0 & 0 & 0 & 0 & 0 & 0 \\
\breve{\phi}_{1} \breve{\kappa}_{1} & \breve{\phi}_{1} \breve{\kappa}_{1} & 0 & 0 & 0 & 0 & 0 & 0 \\
\breve{\phi}_{1} \breve{\kappa}_{1} & 0 & \breve{\phi}_{1} \breve{\kappa}_{1} & 0 & 0 & 0 & 0 & 0 \\
\breve{\phi}_{1} \widetilde{\kappa}_{1} & \breve{\phi}_{1} \breve{\kappa}_{1} & \breve{\phi}_{1} \breve{\kappa}_{1} & \breve{\phi}_{1} \breve{\kappa}_{1} & 0 & 0 & 0 & 0 \\
\Phi_{2}^{*} & 0 & 0 & 0 & \Phi_{2}^{*} & 0 & 0 & 0 \\
\Phi_{2}^{*} & \Phi_{2}^{*} & 0 & 0 & \Phi_{2}^{*} & \Phi_{2}^{*} & 0 & 0 \\
\Phi_{2}^{*} & 0 & \Phi_{2}^{*} & 0 & \Phi_{2}^{*} & 0 & \Phi_{2}^{*} & 0 \\
\Phi_{2}^{*} & \Phi_{2}^{*} & \Phi_{2}^{*} & \Phi_{2}^{*} & \Phi_{2}^{*} & \Phi_{2}^{*} & \Phi_{2}^{*} & \Phi_{2}^{*}
\end{array}\right)\left(\begin{array}{c}
x_{0 u} \\
x_{1 u} \\
x_{2 u} \\
x_{3 u} \\
x_{4 u} \\
x_{5 u} \\
x_{6 u} \\
x_{7 u}
\end{array}\right)+e
$$

where $\Phi_{2}=f_{\epsilon}^{\tau_{2}} \breve{\phi}_{2} \breve{\kappa}_{2}, \Phi_{2}^{*}=\left(f_{\epsilon}^{\tau_{2}} \breve{\phi}_{2} \breve{\kappa}_{2}\right)^{*}, e_{0}$ and $e$ are the polarized noises given by

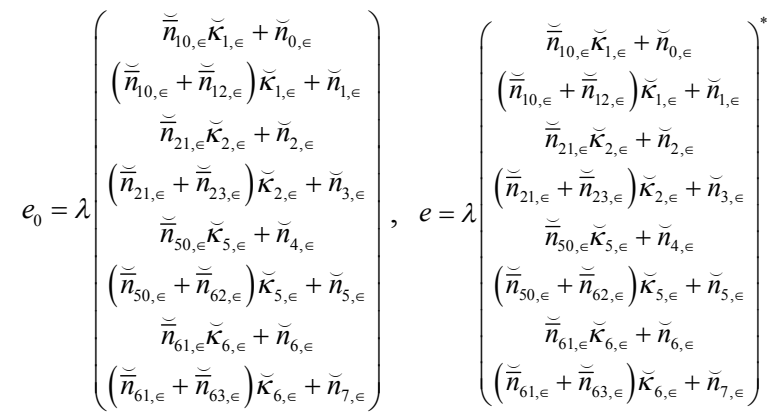

$f_{\epsilon}^{\tau_{2}}=\exp (-12 \pi \in \tau / N), \quad x_{i \in}$ is the $\epsilon^{\text {th }}$ element of $x_{i}$, $\breve{\kappa}_{k, \in}$ is the $\epsilon^{t h}$ element of $\breve{\kappa}_{k}, \overline{\bar{n}}_{k i}$ is the $\epsilon^{t h}$ element of $\breve{n}_{i}$, $\forall k \in\{1, \cdots, 8\}$ and $\forall i \in Z_{8}$.

From equation (19), we can easily see that the channel coefficient is changed to 8 by 8 polar coding. By getting this signal, we can directly decode with a SIC recursive decoding method at the destination.

\section{4-2 The Down Polar Relay Case}

As we mentioned in the previous up-polar relay section, we can finally get an 8 by 8 up-polar signal at the destination. In order to get the 8 by 8 down-polar 
code, another mapping is specified from 2 by 2 downpolar code.

Therefore, we also use the same method for downpolar relay. For the down-polar relay case, we also define another mapping:

$$
\begin{aligned}
& u_{0}=x_{7}, \\
& u_{1}=x_{7}+x_{6}, \\
& u_{2}=x_{5}, \\
& u_{3}=x_{5}+x_{4}, \\
& u_{4}=x_{7}+x_{3}, \\
& u_{5}=x_{6}+x_{2}, \\
& u_{6}=x_{5}+x_{1}, \\
& u_{7}=x_{4}+x_{0} .
\end{aligned}
$$

Therefore, the formulas in (16) can be written in the polar code form on each subcarrier $d, \forall d \in Z_{N}$, as follows:

$$
\left(\begin{array}{l}
y_{0 d} \\
y_{1 d} \\
y_{2 d} \\
y_{3 d} \\
y_{4 d} \\
y_{5 d} \\
y_{6 d} \\
y_{7 d}
\end{array}\right)=\left(\begin{array}{cccccccc}
\breve{\phi}_{1} \widetilde{\kappa}_{1} & 0 & 0 & 0 & 0 & 0 & 0 & 0 \\
0 & \breve{\phi}_{1} \breve{\kappa}_{1} & 0 & 0 & 0 & 0 & 0 & 0 \\
0 & 0 & \breve{\phi}_{1} \breve{\kappa}_{1} & 0 & 0 & 0 & 0 & 0 \\
0 & 0 & 0 & \breve{\phi}_{1} \breve{\kappa}_{1} & 0 & 0 & 0 & 0 \\
0 & 0 & 0 & 0 & \Phi_{2} & 0 & 0 & 0 \\
0 & 0 & 0 & 0 & 0 & \Phi_{2} & 0 & 0 \\
0 & 0 & 0 & 0 & 0 & 0 & \Phi_{2} & 0 \\
0 & 0 & 0 & 0 & 0 & 0 & 0 & \Phi_{2}
\end{array}\right)\left(\begin{array}{c}
u_{4}^{*}+u_{5}^{*}+u_{6}^{*}+u_{7}^{*} \\
u_{4}^{*}+u_{6}^{*} \\
u_{4}^{*}+u_{5}^{*} \\
u_{4}^{*} \\
u_{1}+u_{3} \\
u_{0}+u_{2} \\
u_{1} \\
u_{0}
\end{array}\right)+e_{0}
$$

which can be rewritten as:

$$
\left(\begin{array}{l}
y_{0 d}^{*} \\
y_{1 d}^{*} \\
y_{2 d}^{*} \\
y_{3 d}^{*} \\
y_{4 d} \\
y_{5 d} \\
y_{6 d} \\
y_{7 d}
\end{array}\right)=\left(\begin{array}{cccccccc}
\breve{\phi}_{1} \breve{\kappa}_{1} & \breve{\phi}_{1} \breve{\kappa}_{1} & 0 \breve{\phi}_{1} \breve{\kappa}_{1} & \breve{\phi}_{1} \breve{\kappa}_{1} & \breve{\phi}_{1} \breve{\kappa}_{1} & \breve{\phi}_{1} \breve{\kappa}_{1} & \breve{\phi}_{1} \breve{\kappa}_{1} & \breve{\phi}_{1} \breve{1}_{1} \\
0 & \breve{\phi}_{1} \breve{\kappa}_{1} & 0 & \breve{\phi}_{1} \breve{\kappa}_{1} & 0 & \breve{\phi}_{1} \breve{\kappa}_{1} & 0 & \breve{\phi}_{1} \breve{\kappa}_{1} \\
0 & 0 & \breve{\phi}_{1} \breve{\kappa}_{1} & \breve{\phi}_{1} \breve{\kappa}_{1} & 0 & 0 & \breve{\phi}_{1} \breve{\kappa}_{1} & \breve{\phi}_{1} \breve{\kappa}_{1} \\
0 & 0 & 0 & \breve{\phi}_{1} \breve{\kappa}_{1} & 0 & 0 & 0 & \breve{\phi}_{1} \breve{\kappa}_{1} \\
0 & 0 & 0 & 0 & \Phi_{2}^{*} & \Phi_{2}^{*} & \Phi_{2}^{*} & \Phi_{2}^{*} \\
0 & 0 & 0 & 0 & 0 & \Phi_{2}^{*} & 0 & \Phi_{2}^{*} \\
0 & 0 & 0 & 0 & 0 & 0 & \Phi_{2}^{*} & \Phi_{2}^{*} \\
0 & 0 & 0 & 0 & 0 & 0 & 0 & \Phi_{2}^{*}
\end{array}\right)\left(\begin{array}{l}
x_{0 u} \\
x_{1 u} \\
x_{2 u} \\
x_{3 u} \\
x_{4 u} \\
x_{5 u} \\
x_{6 u} \\
x_{7 u}
\end{array}\right)+e
$$

where $\Phi_{2}=f_{\epsilon}^{\tau_{2}} \breve{\phi}_{2} \breve{\kappa}_{2}, \Phi_{2}^{*}=\left(f_{\epsilon}^{\tau_{2}} \breve{\phi}_{2} \breve{\kappa}_{2}\right)^{*}, e_{0}$ and $e$ are the polarized noises given by:

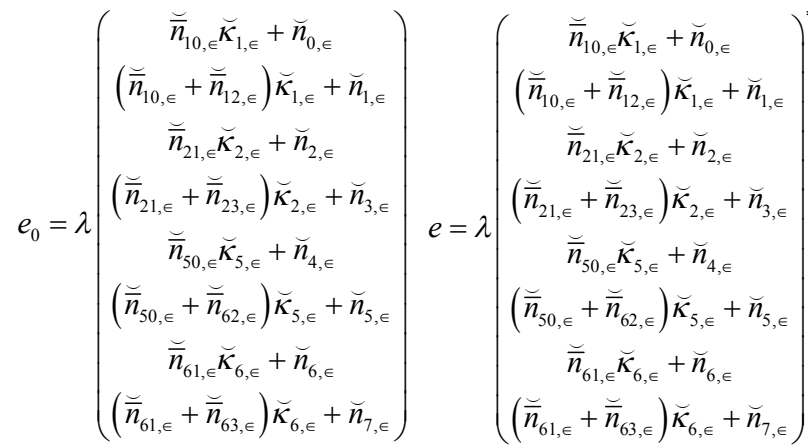

$f_{\epsilon}^{\tau_{2}}=\exp (-12 \pi \in \tau / N), \quad x_{i \in}$ is the $\epsilon^{\text {th }}$ element of $x_{i}$, $\breve{\kappa}_{k, \epsilon}$ is the $\epsilon^{\text {th }}$ element of $\breve{\kappa}_{k}, \breve{n}_{k i}$ is the $\epsilon^{\text {th }}$ element of $\breve{\bar{n}}_{i}$, $\forall k \in\{1, \cdots, 8\}$ and $\forall i \in Z_{8}$. From (19) and (23), we can easily see the up and down 8 polar switching forms, and also, the up-down switch analysis is proved in Appendix A.

\section{Decoding of the Polar Relay System}

In this subsection, we consider the successive interference cancellation (SIC) decoding for the proposed polar MIMO relay system with standard complex constellations, such as binary phase shift keying (BPSK) modulation constellation. Recall that each signal $x_{i, \varepsilon}, \forall \varepsilon$ $\in Z_{N}$, in the $\varepsilon^{\text {th }}$ subcarrier of OFDM block $x_{i}, i \in Z_{8}$, is independently transmitted across $\mathrm{R}_{\mathrm{k}}$ and a channel output $y_{i, \varepsilon}$ is obtained with transition probability $W\left(y_{i, \varepsilon} \mid x_{i, \varepsilon}\right)$. For each subcarrier in eight OFDM symbols we misuse the simplified notations $\mathrm{x}_{=} \mathrm{x}_{\varepsilon}=\left(\mathrm{x}_{I, \varepsilon}^{T}, \mathrm{x}_{F, \varepsilon}^{T}\right)^{T}, \mathrm{y}=\mathrm{y}_{\varepsilon}=\left(\mathrm{y}_{1, \varepsilon}^{T}\right.$, $\left.\mathrm{y}_{2, \varepsilon}^{T}\right)^{T}$ for the up-down polarizing system, where $\mathrm{y}_{1}=\mathrm{y}_{1, \varepsilon}$ $=\left(y_{0, \varepsilon}, y_{1, \varepsilon}\right)^{T}$ and $\mathrm{y}_{2}=\mathrm{y}_{2, \varepsilon}=\left(y_{2, \varepsilon}, y_{3, \varepsilon}\right)^{T}$.

We consider all single links of the relay channel $\mathrm{H}$ from each pair of transmit antenna of source node $\mathrm{S}$ and receive antenna of relay node $R_{k}$, and $K$ from relay node $\mathrm{R}_{\mathrm{k}}$ to destination node $\mathrm{D}$, which are independent complex Gaussian random variables with zero-mean and unitvariance. Each single-link channel, denoted by $\mathrm{W}$, has the transition probability $W(y \mid x)$, where $x, y \in \mathrm{A}$. As a useful measurement of the reliability of the wireless network, a conventional channel parameter, the symmetric capacity $\mathrm{I}(\mathrm{W})$, can be used with some modulations. Note that parameter I(W) is the highest rate at which the reliable communication is possible using inputs with equal probabilities.

The successive iterative cancellation (SIC) decoder of the down-polarizing system observes $y$ and generates an estimate $\hat{x}$ of $x$. We may visualize the decoder as consisting of four decision elements, each element $\hat{\mathrm{x}}_{i}$ for source element $x_{i}, \forall \in Z_{8}$.

The depolarizing algorithm of the polar relay system begins with the $i^{\text {th }}$ decision element $\hat{x}_{i}$ for the downpolarizing system. It waits until it has received all previous decisions $\hat{x}_{i-1}$, and upon receiving them, it calculates the likelihood ratio (LR) $L_{8}^{(i)}$ as follows:

$$
L_{8}^{(i)}\left(\mathrm{y}, \hat{x}_{1} \cdots \hat{x}_{i-1}\right)=\frac{W_{8}^{(i)}\left(\mathrm{y}, \hat{x}_{1} \cdots \hat{x}_{i-1} \mid 0\right)}{W_{8}^{(i)}\left(\mathrm{y}, \hat{x}_{1} \cdots \hat{x}_{i-1} \mid 1\right)},
$$

and generates its decision as: 


$$
\hat{x}_{i}=\left\{\begin{array}{cc}
0, & \text { if } L_{8}^{(i)}\left(\mathrm{y}, \hat{x}_{1} \cdots \hat{x}_{i-1}\right) \geq 1, \\
1, & \text { otherwise }
\end{array}\right.
$$

which is then sent to succeeding decision element $\hat{x}_{i+1}$. The proof of (25) is found in Appendix B. The complexity of the decoding algorithm is determined essentially by the complexity of calculating LRs, which is $\mathrm{N}(1+\log 2 \mathrm{~N})=32$ for computing one round.

However, for the up-polarizing system the low level of LRs are given in [1] by

$$
\begin{aligned}
& L_{2}^{\prime(0)}\left(\mathrm{y}_{k}\right)=\frac{L\left(y_{2 k-2}\right) L\left(y_{2 k-1}\right)+1}{L\left(y_{2 k-2}\right)+L\left(y_{2 k-1}\right)} \\
& L_{2}^{\prime(1)}\left(y_{k}, \hat{x}_{2 k-2}\right)=\left(L\left(y_{2 k-2}\right)\right)^{1-2 \hat{x}_{2 k-2}} L\left(y_{2 k-1}\right),
\end{aligned}
$$

Furthermore, the high level of LRs can be calculated as

$$
\begin{aligned}
& L_{8}^{\prime(0)}(\mathrm{y})=\frac{L_{4}^{\prime(0)}\left(y_{1}\right) L_{4}^{(0)}\left(y_{2}\right)+1}{L_{4}^{(0)}\left(y_{1}\right)+L_{4}^{(0)}\left(y_{2}\right)} \\
& L_{8}^{\prime(1)}\left(y_{0}, \hat{\mathrm{x}}_{0}\right)=\left(L_{4}^{\prime(0)}\left(y_{1}\right)\right)^{1-2 \hat{x}_{0}} L_{4}^{\prime(0)}\left(y_{2}\right), \\
& L_{8}^{\prime(2)}\left(\mathrm{y}, \hat{\mathrm{x}}_{F}\right)=\frac{L_{4}^{\prime(1)}\left(y_{1}, \hat{x}_{0}\right) L_{4}^{\prime(1)}\left(y_{2}, \hat{x}_{0}+\hat{x}_{1}\right)+1}{L_{4}^{(1)}\left(y_{1}, \hat{x}_{0}\right)+L_{4}^{\prime(1)}\left(y_{2}, \hat{x}_{0}+\hat{x}_{1}\right)} \\
& L_{8}^{\prime(3)}\left(y_{0}, \hat{\mathrm{x}}_{0}\right)=L_{4}^{\prime(1)}\left(y_{1}, \hat{x}_{0}\right)^{1-2 \hat{x}_{2}} L_{4}^{\prime(1)}\left(y_{2}, \hat{x}_{0}+\hat{x}_{1}\right), \\
& L_{8}^{\prime(4)}(\mathrm{y})=\frac{L_{4}^{\prime(2)}\left(y_{1}\right) L_{4}^{\prime(2)}\left(y_{2}\right)+1}{L_{4}^{(2)}\left(y_{1}\right)+L_{4}^{\prime(2)}\left(y_{2}\right)} \\
& L_{8}^{\prime(5)}\left(y_{0}, \hat{\mathrm{x}}_{0}\right)=\left(L_{4}^{(2)}\left(y_{1}\right)\right)^{1-2 \hat{x}_{0}} L_{4}^{\prime(2)}\left(y_{2}\right), \\
& L_{8}^{\prime(6)}\left(\mathrm{y}, \hat{\mathrm{x}}_{F}\right)=\frac{L_{4}^{\prime(3)}\left(y_{1}, \hat{x}_{0}\right) L_{4}^{\prime(3)}\left(y_{2}, \hat{x}_{0}+\hat{x}_{1}\right)+1}{L_{4}^{(3)}\left(y_{1}, \hat{x}_{0}\right)+L_{4}^{(3)}\left(y_{2}, \hat{x}_{0}+\hat{x}_{1}\right)} \\
& L_{8}^{\prime(7)}\left(y_{0}, \hat{\mathrm{x}}_{0}\right)=L_{4}^{\prime(3)}\left(y_{1}, \hat{x}_{0}\right)^{1-2 \hat{x}_{2}} L_{4}^{\prime(3)}\left(y_{2}, \hat{x}_{0}+\hat{x}_{1}\right),
\end{aligned}
$$

Thus far, we have calculated LRs of the polar MIMO OFDM relay system with two polar systems. The advantage of this depolarizing algorithm is the relationship of two level LRs in coordination. For example, two LRs, $L_{4}^{(0)}\left(\mathrm{y}_{t}\right)$ and $L_{4}^{(1)}\left(\mathrm{y}_{t}, \hat{x}_{0}\right)$, are assembled from the same pair of LRs, $L_{2}^{(0)}\left(y_{1}\right)$ and $L_{2}^{(0)}\left(y_{2}\right)$, while the other two LRs, $L_{4}^{(2)}\left(\mathrm{y}_{t}, \hat{\mathrm{x}}_{1}\right)$ and $L_{4}^{(3)}\left(\mathrm{y}_{t}, \hat{\mathrm{x}}_{1} \hat{\mathrm{x}}_{2}\right)$, are from LRs $L_{2}^{(1)}\left(y_{1}, \hat{x}_{0}\right)$ and $L_{2}^{(1)}\left(y_{2}, \hat{x}_{0}+\hat{x}_{1}\right)$ due to the symmetry properties of MIMO relay channels. In addition, two down level LRs $L_{2}^{(0)}\left(y_{k}\right)$ and $L_{2}^{(1)}\left(y_{k}, \hat{x}_{2 k-2}\right)$ are assembled from the lowest (initial) level LRs $L^{(0)}\left(y_{2 k-2}\right)$ and $L^{(0)}\left(y_{2 k-1}\right) \forall k \in\{1,2, \cdots 8\}$. This process proposes an elegant approach for an accurate count of the total number of LRs that are required for a full description of the decoding algorithm.

Next, we design an implementation of the SC decoder for switching the polar MIMO-OFDM relay system. There are 12 nodes corresponding to LRs for decision elements $\hat{x}$.

Note that, in the down-polarizing system, it is not necessary to generate $\hat{x}_{2}$ and $\hat{x}_{3}$ since they are frozen bits

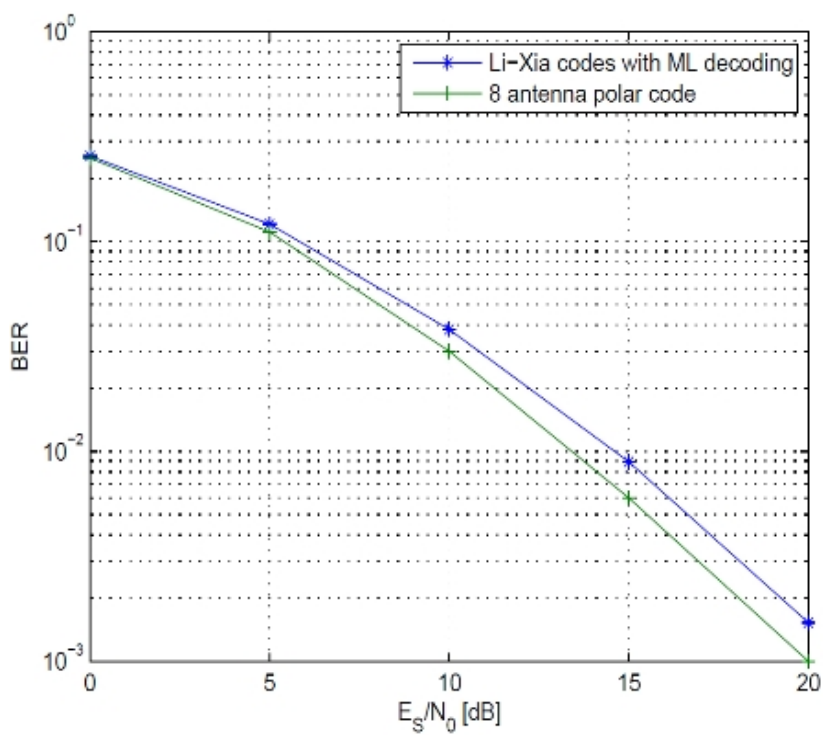

Fig. 5. The polar MIMO-OFDM relay system based on the OFDM polarizing for the FSF channels.

that have low-reliability while transmitting in the downpolarizing channels. Similarly, in the up-polarizing system, it is not necessary to generate $\hat{x}_{0}$ and $\hat{x}_{1}$ since they are frozen bits with the low-reliability while transmitting in the up-polarizing channels. In this way, the proposed depolarizing algorithm can be made while directly setting $\hat{x}_{2}=\hat{x}_{3}=0$ in the down-polarizing system and setting $\hat{x}_{0}=\hat{x}_{1}=0$ in the up-polarizing system. This decoding process continues until all information bits $\mathrm{x}$ are jointly decoded in the end. Next, we will show the BER performance behaviors of the polar MIMO-OFDM relay system with simulation results. Thus, we can obtain $\mathrm{x}$ from $\hat{x}$ in the polar MIMO-OFDM relay system with the high-reliability.

In Fig. 5, compared to the Li-Xia-Lee ML decoding MIMO-OFDM system, we can obtain $1 \mathrm{~dB}$ coding gain with 8 antenna up-down polar coding. In the proposed scheme, we do not require relays to decode. Only a simple encoding operation is done at each relay, which makes the transmission very simple and hence we can avoid imposing bottlenecks on the data rate. The complexity is then lower than that reported in [8].

\section{Conclusion}

In this paper, we have presented a simple design for a cooperative relay scheme based on the 8 antenna polar relay switching system with two polarizing systems, which are shown as equation (19) and (23), the up-down polarizing system for full diversity. Two polar coding processes are available for each polarizing system, in- 
cluding source polarizing and relay polarizing sequences. The present polarizing relay system has a salient recursiveness feature and can be decoded with a SIC decoder, which renders the scheme analytically tractable and provides a low-complexity coding algorithm while multiple antennas are equipped. We analyzed the BER performance and diversity of such systems based on the Space Time Coding (STC) codes with the fixed size using the polarizing channels, which tend to polarize with respect to the increasing reliability under certain channel combining and splitting operations. Using this method, we achieved maximum diversity gains based on switching the state polar code with information bits. Each relay node only needs a simple processing operation to select and retransmit the partial signals without requiring any contemplation to decode the noisy signals. Simulation shows that the present scheme can provide an alternative solution for transmitting with higher reliability than the conventional relay schemes due to the occurrence of capacity-achieving OFDM sequences. This demonstrates that the proposed polar relay system has similar BER performance behaviors to the STC codes, but outperforms the STC codes in terms of the BER performance for large transmission power when the depolarizing algorithm is applied at the receiver.

\section{Appendix}

\section{A. Proof of the Up-down Switch Analysis}

From (11), we consider the following binary matrices in $\mathrm{GF}(2)$ :

$$
Q_{2}=\left(\begin{array}{ll}
1 & 0 \\
1 & 1
\end{array}\right), Q_{2}^{T}=\left(\begin{array}{ll}
1 & 1 \\
0 & 1
\end{array}\right)
$$

It can be easily checked that

$$
\begin{aligned}
& Q_{2}^{2}=\left(\begin{array}{ll}
1 & 0 \\
1 & 1
\end{array}\right) \times\left(\begin{array}{ll}
1 & 0 \\
1 & 1
\end{array}\right)=\left(\begin{array}{ll}
1 & 0 \\
0 & 1
\end{array}\right)=I_{2}=\left(Q_{2}^{T}\right)^{2} \\
& Q_{2} Q_{2}^{T}=\left(\begin{array}{ll}
1 & 0 \\
1 & 1
\end{array}\right) \times\left(\begin{array}{ll}
1 & 1 \\
0 & 1
\end{array}\right)+\left(\begin{array}{ll}
1 & 1 \\
0 & 1
\end{array}\right) \times\left(\begin{array}{ll}
1 & 0 \\
1 & 1
\end{array}\right) \\
&=\left(\begin{array}{ll}
1 & 1 \\
1 & 0
\end{array}\right)+\left(\begin{array}{ll}
0 & 1 \\
1 & 1
\end{array}\right)=I_{2}
\end{aligned}
$$

Furthermore,

$$
\left(\begin{array}{cc}
Q_{2} & Q_{2}^{T} \\
Q_{2}^{T} & \mathrm{Q}_{2}
\end{array}\right) \times\left(\begin{array}{ll}
Q_{2}^{T} & Q_{2} \\
Q_{2} & Q_{2}^{T}
\end{array}\right)=\left(\begin{array}{cccc}
1 & 0 & 0 & 0 \\
0 & 1 & 0 & 0 \\
0 & 0 & 1 & 0 \\
0 & 0 & 0 & 1
\end{array}\right) .
$$

Now we present a 4 by 4 polar matrix based on submatrix $\alpha$ and $\beta$ defined. Let

$$
Q_{4}=\left(\begin{array}{ll}
Q_{2} & Q_{2}^{T} \\
Q_{2}^{T} & Q_{2}
\end{array}\right)=\left(\begin{array}{llll}
1 & 0 & 1 & 1 \\
1 & 1 & 0 & 1 \\
1 & 1 & 1 & 0 \\
0 & 1 & 1 & 1
\end{array}\right) .
$$

We have

$$
Q_{4}^{-1}=\left(\begin{array}{ll}
Q_{2}^{T} & Q_{2} \\
Q_{2} & Q_{2}^{T}
\end{array}\right)=\left(\begin{array}{llll}
1 & 0 & 1 & 1 \\
1 & 1 & 0 & 1 \\
1 & 1 & 1 & 0 \\
0 & 1 & 1 & 1
\end{array}\right)^{T}
$$

then

$$
Q_{4} Q_{4}^{-1}=I_{4} .
$$

Generator matrix $Q_{N}, N=2^{n}$ is shown:

$$
\begin{aligned}
Q_{N} & =\left(Q_{\frac{N}{2}} \otimes Q_{2}\right) \\
& =\left(I_{\frac{N}{2}} \otimes Q_{2}\right)\left(Q_{\frac{N}{2}} \otimes I_{2}\right) \\
& =\prod_{i=0}^{n}\left(I_{\frac{N}{2}} \otimes Q_{2} \otimes I_{2^{(i-1)}}\right) \\
& =\prod_{i=0}^{n}\left(Q_{N}^{i}\right) .
\end{aligned}
$$

where $\otimes$ is defined as Kronecker product.

For example, $N=2^{2}$ case:

$$
\begin{aligned}
Q_{4} & =\left(\begin{array}{ll}
1 & 0 \\
1 & 1
\end{array}\right) \otimes\left(\begin{array}{ll}
1 & 0 \\
1 & 1
\end{array}\right)=\left(I_{2} \otimes Q_{2}\right)\left(Q_{2} \otimes I_{2}\right) \\
& =\left[\left(\begin{array}{ll}
1 & 0 \\
0 & 1
\end{array}\right) \otimes\left(\begin{array}{ll}
1 & 0 \\
1 & 1
\end{array}\right)\right]\left[\left(\begin{array}{ll}
1 & 0 \\
1 & 1
\end{array}\right) \otimes\left(\begin{array}{ll}
1 & 0 \\
0 & 1
\end{array}\right)\right] \\
& =\left(\begin{array}{llll}
1 & 0 & 0 & 0 \\
1 & 1 & 0 & 0 \\
1 & 0 & 1 & 0 \\
1 & 1 & 1 & 1
\end{array}\right)
\end{aligned}
$$

We have, $N=2^{3}$ case:

$$
\begin{aligned}
Q_{8} & =\prod_{i=1}^{3}\left(I_{2^{3-i}} \otimes Q_{2} \otimes I_{2^{(i-1)}}\right) \\
& =\left(I_{4} \otimes Q_{2} \otimes I_{1}\right)\left(I_{2} \otimes Q_{2} \otimes I_{2}\right)\left(I_{1} \otimes Q_{2} \otimes I_{4}\right) \\
& \left(\begin{array}{lllllllll}
1 & 0 & 0 & 0 & 0 & 0 & 0 & 0 \\
1 & 1 & 0 & 0 & 0 & 0 & 0 & 0 \\
1 & 0 & 1 & 0 & 0 & 0 & 0 & 0 \\
1 & 1 & 1 & 1 & 0 & 0 & 0 & 0 \\
1 & 0 & 0 & 0 & 1 & 0 & 0 & 0 \\
1 & 1 & 0 & 0 & 1 & 1 & 0 & 0 \\
1 & 0 & 1 & 0 & 1 & 0 & 1 & 0 \\
1 & 1 & 1 & 1 & 1 & 1 & 1 & 1
\end{array}\right)
\end{aligned}
$$




\section{B. SC (Successive Cancellation) Recursive Algorithm}

Proof: From (25), we have log-likelihood ratio(LLR) [9], [10].

$$
L(d)=\log _{e}\left[\frac{P(d=0)}{P(d=1)}\right]=\log _{e}\left[\frac{P(d=0)}{1-P(d=0)}\right]
$$

we can see that

$$
\begin{aligned}
& e^{L(d)}=\left[\frac{P(d=0)}{1-P(d=0)}\right] \\
& e^{L(d)}-e^{L(d)} P(d=0)=P(d=0) \\
& P(d=0)\left[1+e^{L(d)}\right]=e^{L(d)} \\
& P(d=0)=\frac{e^{L(d)}}{1+e^{L(d)}} \\
& P(d=1)=1-P(d=0)=1-\frac{e^{L(d)}}{1+e^{L(d)}}=\frac{1}{1+e^{L(d)}} \\
& L\left(d_{1} \oplus d_{2}\right)= \\
& \log _{e}\left[\frac{P\left(d_{1}=0\right) P\left(d_{1}=1\right)+\left(1-P\left(d_{1}=0\right)\right)\left(1-P\left(d_{2}=1\right)\right)}{P\left(d_{1}=0\right) P\left(d_{1}=0\right)+\left(1-P\left(d_{1}=0\right)\right)\left(1-P\left(d_{2}=0\right)\right)}\right] \\
& =\log _{e}\left[\frac{\left(\frac{e^{L\left(d_{1}\right)}}{1+e^{L\left(d_{1}\right)}}\right)\left(\frac{1}{1+e^{L\left(d_{2}\right)}}\right)+\left(\frac{1}{1+e^{L\left(d_{1}\right)}}\right)\left(\frac{e^{L\left(d_{2}\right)}}{1+e^{L(d 2)}}\right)}{\left.\left(\frac{e^{L\left(d_{1}\right)}}{1+e^{L\left(d_{1}\right)}}\right)\left(\frac{e^{L\left(d_{2}\right)}}{1+e^{L\left(d_{2}\right)}}\right)+\left(\frac{1}{1+e^{L\left(d_{1}\right)}}\right)\left(\frac{1}{1+e^{L(d 2)}}\right)\right]}\right. \\
& =\log _{e}\left[\frac{e^{L\left(d_{1}\right)}+e^{L\left(d_{2}\right)}}{\left(1+e^{L\left(d_{1}\right)}\right)\left(1+e^{L\left(d_{2}\right)}\right)}\right] \\
& \left.\frac{e^{L\left(d_{1}\right)} e^{L\left(d_{2}\right)}+1}{\left.1+e^{L\left(d_{1}\right)}\right)\left(1+e^{L\left(d_{2}\right)}\right)}\right]
\end{aligned}
$$

where the $\oplus$ sign is used to denote the modulo- 2 sum of data expressed as binary digits. The $\Leftrightarrow$ denotes $\log$ likelihood addition.

This gives:

$$
\begin{aligned}
& \left(L\left(d_{1}\right) \Leftrightarrow L\left(d_{2}\right)\right)=L\left(d_{1} \oplus d_{2}\right)=\log _{e} \frac{e^{L\left(d_{1}\right)}+e^{L\left(d_{2}\right)}}{e^{L\left(d_{1}\right)} e^{L\left(d_{2}\right)}+1} \\
& e^{L\left(d_{1} \oplus d_{2}\right)}=\frac{e^{L\left(d_{1}\right)}+e^{L\left(d_{2}\right)}}{e^{L\left(d_{1}\right)} e^{L\left(d_{2}\right)}+1} \\
& \left(e^{L\left(d_{1} \oplus d_{2}\right)}\right)^{-1}=\frac{e^{L\left(d_{1}\right)} e^{L\left(d_{2}\right)}+1}{e^{L\left(d_{1}\right)}+e^{L\left(d_{2}\right)}}
\end{aligned}
$$

Defining $e^{L\left(d_{1}\right)}, e^{L\left(d_{2}\right)},\left(e^{L\left(d_{1} \oplus d_{2}\right)}\right)^{-1}$ as follows:

$$
\begin{aligned}
& e^{L\left(d_{1}\right)}=L_{N / 2}^{(i)}\left(y_{1}^{N / 2}, \hat{u}_{1,0}^{2 i-2}\right), \\
& e^{L\left(d_{2}\right)}=L_{N / 2}^{(i)}\left(y_{N / 2+1}^{N / 2}, \hat{u}_{1, e}^{2 i-2}\right),
\end{aligned}
$$

$$
\left(e^{L\left(d_{1} \oplus d_{2}\right)}\right)^{-1}=L_{N / 2}^{(2 i-1)}\left(y_{1}^{N}, \hat{u}_{1}^{2 i-2}\right),
$$

Finally, the recursive decoding is specified as:

$$
\begin{aligned}
& L_{N / 2}^{2 i-1}\left(y_{1}^{N}, \hat{u}_{1}^{2 i-2}\right)= \\
& \quad \frac{1+L_{N / 2}^{(i)}\left(y_{1}^{N / 2}, \hat{u}_{1 . o}^{2 i-2} \oplus \hat{u}_{1, e}^{2 i-2}\right) \cdot L_{N / 2}^{(i)}\left(y_{N / 2+1}^{N}, \hat{u}_{1 . e}^{2 i-1}\right)}{L_{N / 2}^{(i)}\left(y_{1}^{N / 2}, \hat{u}_{1 . o}^{2 i-2} \oplus \hat{u}_{1, e}^{2 i-2}\right)+L_{N / 2}^{(i)}\left(y_{N / 2+1}^{N}, \hat{u}_{1 . e}^{2 i-1}\right)}
\end{aligned}
$$

From equation (52), we can easily generate the 8 polar decoding formula (28).

This work was supported by World Class University R32-2010-000-20014-0, Fundamental Research 20100020942, and the second stage of Brain Korea 21 Project in 2011, National Research Foundation (NRF), Republic of Korea.

\section{References}

[1] E. Arikan, "Channel polarization: A method for constructing capacity-achieving codes for symmetric binary-input memoryless channel", IEEE Trans. Inf. Theory, vol. 55, pp. 4366-4385, Jul. 2009.

[2] Y. Guo, M. H. Lee, "A novel channel polarization on binary discrete memoryless channels", IEEE ICCS, Singapore, Nov. 2010.

[3] H. Jafarkhani, "Space-time-state block coded MIMO communication systems using reconfigurable antennas", IEEE Trans. Commun., vol. 8, pp. 6019-6029, Dec. 2009

[4] S. M. Alamouti, "A simple transmit diversity technique for wireless communications", IEEE J. Sel. Areas Commun., vol. 16, pp. 1451-1458, Oct. 1998.

[5] H. Jafarkhani, "A quasi-orthogonal space-time block codes", IEEE Trans. Commun., vol. 49, pp. 1-4, Jan. 2001.

[6] W. Su, X.-G. Xia, and K. J. R. Liu, "A systematic design of high-rate complex orthogonal space-time block codes", IEEE Trans. Inf. Theory, vol. 8, pp. 4340-4347, Jun. 2004.

[7] Y. Shang, X.-G. Xia, "Space-time block codes achieving full diversity with linear receivers", IEEE Trans. Inf. Theory, vol. 54, pp. 4528-4547, Oct. 2008.

[8] Z. Li, X.-G. Xia, and Moon Ho Lee, "A simple orthogonal space-time coding scheme for asynchronous cooperative systems for frequency selective fading channels", IEEE Trans. Commun., vol. 58, pp. 2219-2224, Aug. 2010.

[9] Moon Ho Lee, Eun Ji Choi, Jae Seung Yang, and Ju Yong Park, "Encoding and decoding of radix 4 polar code", The Institute of Electronics Engineers of Ko- 
rea, vol. 46, Tc, no. 10, Oct. 2009.

[10] Moon Ho Lee, Jun Li, and Ju Yong Park, "Successive cancellation decoding of polar coding channel

Jun Li

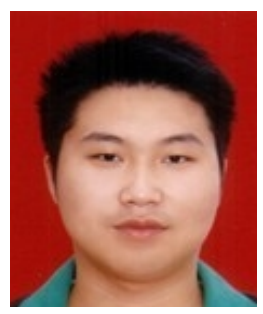

received his B.S. degree in electronic engineering from South Central University for Nationalities, P.R. China, in 2009, and his M.S. degree in electronic engineering from Chonbuk National University, Jeonju, Republic of Korea, in 2011. He has been doing research on the digital mobile wireless communications based on polar codes, MIMO-OFDM since September 2011 participating in the World Class University (WCU) project sponsored by the National Research Foundation (NRF) as a researcher studying for his doctorate who is presently affiliated to the Institute of Information and Communications in Chonbuk National University. synthesis and decomposition", The Institute of Electronics Engineers of Korea, vol. 48, Tc, no. 14, Apr. 2011.

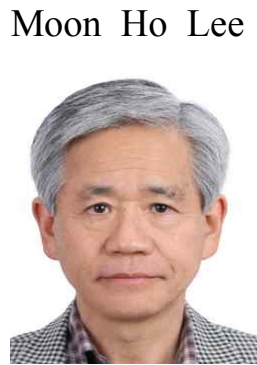

received both his B.S. and M.S. degrees in electrical engineering from Chonbuk National University, Jeonju, Republic of Korea, in 1967 and 1976, respectively, and his Ph.D. degrees in electronics engineering from Chonnam National University, Gwangju, Republic of Korea, in 1984 and from the University of Tokyo, Japan, in 1990, respectively. From 1970 to 1980, he was a chief engineer with Namyang Moonhwa Broadcasting Corporation. Since 1980, he has been a professor in the department of electronics engineering at Chonbuk National University. From 1985 to 1986, he was also with the University of Minnesota, as a postdoctoral fellow. He held visiting positions at the University of Hanover, Germany, in 1990, the University of Aachen, Germany in 1992 and 1996, respectively, and the University of Munich, Germany, in 1998. He has authored 34 books, including Digital Communication (Youngil, 1999, Republic of Korea), Information and Coding (Bokdu, 1998, Republic of Korea), Digital Image Processing (Daeyoung, 1994, Republic of Korea), and Digital Filter Design (Daeyoung, 1995, Republic of Korea). His current research interests include multi-dimensional source and channel coding, mobile communications, image processing, information security, signal processing, digital communications, and polar codes. He is a Registered Telecommunication Professional Engineer and a member of the National Academy of Engineering in Korea. He was the recipient of the paper prize awards from the Korean Institute of Communication Science in 1986 as well as in 1997, the Institute of Electronics Engineers of Korea in 1987, Chonbuk Province in 1992, and the commendation of the Prime Minister in 2002 for inventing the Jacket matrix, respectively. In addition, he won the Best Paper Awards from ICSEA, France, in 2006, ICSNC, France, in 2006, and EITC, Republic of Korea, in 2006, respectively. Furthermore, he won the National Order of Merit, the Doyak Medal, from the Korean government in 2007, the Haedong Information and Communications Best Prize from the Korea Information and Communications Society in 2007, the Award for Scientist and Engineer of the Month from the Ministry of Science and Technology and the Korea Science and Engineering Foundation in January, 2008, the Best Paper Awards from CEIC 2009 and CEIC 2010 sponsored by IEEE's Korea Daejeon Section, and the Grand Alumni Award from the Chonbuk National University's alumni association in 2009, respectively. Moreover, he has been a principal of the World Class University project since 2008 and an honor professor of Central South University, P. R. China, since 2009. 
Yier Yan

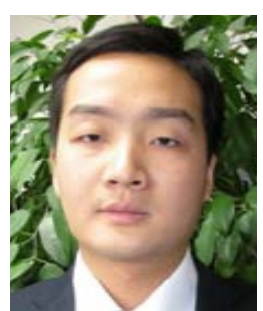

received the B.S. degree of Applied Electronic Engineering from South Central University for Nationalities, Wuhan, Hubei, P. R. China, in 2004. He received both his M.S. degree of communication engineering and $\mathrm{Ph} . \mathrm{D}$. of electronic engineering from Chonbuk National University, Jeonju, Republic of Korea, in 2006 and 2010, respectively. He has been a professor in the Mechanical and electronic school of Guangzhou University, Guangzhou, P. R. China, since 2010. His research interests include information theory, signal processing, and OFDM in MIMO system.

\section{Bu Shi Peng}

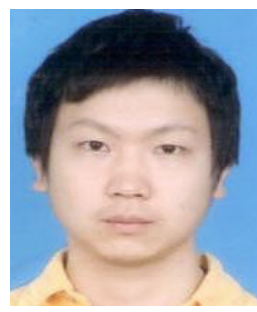

received his B.S. degree of Electrical Engineering from Guizhou University, Guiyang, P. R. China, in 2009. He is currently affiliated with the Institute of Information \& Communications as a researcher toward M. S. degree, in Chonbuk National University, Jeonju, Republic of Korea. And he has been doing research on wireless mobile communications. His main research interests are MIMO, OFDM, ECC, and network coding.

\section{Gun Joon Hwang}

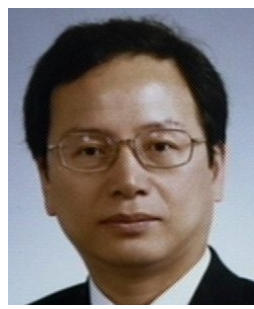

received the B.S. degree from the Division of Information and Telecommunication Engineering, Howon University, Kunsan, Republic of Korea, in 1999. He received his M.S degree of Department of Information Science from Chonbuk National University, Jeonju, Republic of Korea in 2001. He completed his doctoral coursework in 2005, and he has been still doing research toward $\mathrm{PhD}$ degree. He is currently a chief of supporting center of Information and Communication, in Jeonju University, Jeonju, Republic of Korea. His interests areas include internet website design, and software design. 